\title{
HOMOTOPY THEORIES FOR DIAGRAMS OF SPACES
}

\author{
E. DROR FARJOUN
}

\begin{abstract}
We show that the category of diagrams of topological spaces (or simplicial sets) admits many interesting model category structures in the sense of Quillen [8]. The strongest one renders any diagram of simplicial complexes and simplicial maps between them both fibrant and cofibrant. Namely, homotopy invertible maps between such are the weak equivalences and they are detectable by the "spaces of fixed points." We use a generalization of the method for defining model category structure of simplicial category given in [5].
\end{abstract}

0. The main results. In [5 and 6] D. Kan and W. Dwyer give a classification theory for diagrams of simplicial sets and define a model category structure for such diagrams. Similar structures are discussed also by [7]. In their work [6] $D$-diagrams are classified up to a certain weak equivalence called here "objectwise weak equivalence" (see below). This same equivalence plays the role of weak equivalence in the sense of Quillen, in their model category structure.

The main aim of the present note is to show that their results can be generalized and applied to yield:

(i) a whole collection of model category structures on diagrams of spaces with much stronger notions of weak equivalence-rendering such equivalences homotopy invertible under much milder assumptions,

(ii) associated with (i), a classification of $D$-diagram up to stronger notions of weak equivalence,

(iii) a Bredon-like result for detecting homotopy invertible maps of diagrams of topological spaces or simplicial sets (compare $[2,4]$ ). For example, in $\operatorname{Top}^{D}$, the category of diagrams of topological spaces, one gets the following direct generalization of Bredon's theorem [2, 5.5]: A map $f:|X| \rightarrow|Y|$ between realization of diagrams of simplicial sets is a weak equivalence if and only if it has a $D$-homotopy inverse $g:|Y| \rightarrow|X|$.

A direction of application for the construction of an equivariant instable Adams spectral sequence for equivariant function complexes is discussed briefly in the last section.

0.1. Types of weak equivalences. Let us now briefly recall the concept of weak equivalence in [5], to which we will refer here as objectwise weak equivalence. A map $f: X \rightarrow Y$ beween two $D$-diagrams (i.e. functors $X, Y: D \rightarrow($ spaces)) is an objectwise weak equivalence if for each object $d \in \operatorname{obj} D$ the map $f(d): X(d) \rightarrow Y(d)$

Received by the editors April 25, 1985 and, in revised form, July 3, 1986.

1980 Mathematics Subject Classification (1985 Revision). Primary 55P91, 55P10; Secondary 55N91.

Key words and phrases. Diagrams of spaces, model category, singular functors, equivariant homotopy theory. 
is a weak equivalence of spaces. (Space $=$ simplicial set or topological spaces). For example, if $D$ is the category with one object associated to a group $G$, whose morphisms are elements of the group, then an objectwise weak equivalence between two $G$-spaces is a $G$-map $f: X \rightarrow Y$ such that, disregarding the $G$-structure, $f$ is a weak homotopy equivalence of spaces. Such an objectwise equivalence has a $G$-homotopy inverse if $X, Y$ are free and fibrant as $G$-spaces. The same holds for any small category $D$.

Ideally, one would like a concept of weak equivalence that will be more restrictive, namely, that would yield a $D$-homotopy inverse under weaker conditions, preferably local conditions, on $X, Y$-namely, conditions on each $X(d)$ or $Y(d)$ as spaces.

In fact, one corollary of our results is the following (see 0.5 ):

0.2. THEOREM. There exists a model category structure of diagrams of topological spaces in which the realization of any diagram of simplicial sets $X: D \rightarrow S$, is cofibrant and fibrant.

Proof. See $\$ 2$ below.

Of course, a weak equivalence between diagrams that are both fibrant and cofibrant is always an (homotopy) equivalence.

0.3. Classes of orbits. Our results are consequences of the technique of [5] using two extra ingredients. To explain these recall that at the heart of the method of [5] is the definition of model category structure using a collection of objects called orbits. The collection $\left\{O_{e}\right\}$ must satisfy certain conditions (see Q0-Q3 in 1.2 below). These orbits are used to define weak equivalences and check certain lifting properties that characterize a model category structure. Our main tool here is to generalize the discussion of orbits in [5] as follows:

First, we do not require that the collection $\left\{O_{e}\right\}$ forms a set. Rather it can be a large class of objects in the model category as long as this class is locally small in the sense of 1.1 below.

Second, we show that there are much larger collections of objects in $S^{D}$ or Top ${ }^{D}$ : for a small diagram $D$, that satisfy the axioms of orbits. This then gives stronger model category structures on $S^{D}$ than $[5,1.3]$.

0.4. Examples of model category structures. Here we outline several examples of model category structures that can be gotten using the generalization of the concept of set of orbits discussed above. They follow as in $\$ 2$ from 1.3 below.

0.5. Orbits for diagrams of spaces. It is well known that the equivariant homotopy theory of $G$-spaces is captured by declaring a map $f: X \rightarrow Y$ to be a weak equivalence if $f$ induces a weak equivalence

$$
f^{e}: \operatorname{hom}\left(O_{e}, X\right) \rightarrow \operatorname{hom}\left(O_{e}, Y\right)
$$

of space for every $G$-orbit

$$
O_{e} \cong G / H \quad(H \subseteq G)
$$

It turns out that the natural generalization of $G$-orbit to " $D$-orbit" for any small category $D$ is a $D$-diagram $T$ whose colimit over $D$ is the one point space. Thus Theorem 0.2 above is proven by defining a map $f: X \rightarrow Y$ in $S^{D}$ to be a weak 
equivalence if $f^{T}: \operatorname{hom}(T, X) \rightarrow \operatorname{hom}(T, Y)$ is a weak equivalence in $S$ for every $D$-orbit. However, the moment $D$ is not a groupoid the collection of $D$-orbits in this sense is not a set. Example: Take $D$ to be the category $(\cdot \rightarrow \cdot)$ with two objects and one nonidentity map between them. Nonetheless, we show that the collection of all $D$-orbits is locally small (1.1) and satisfies axioms Q0-Q3 below and therefore can be used to define a model category structure on $S^{D}$. This structure is the direct generalization to arbitrary small categories of the usual equivariant structure on $G$-space when all subgroups $H \subseteq G$ of $G$ are taken into account [5, 1.2]. Further, as in $[5,1.7]$, one may choose other collections of orbits and thus get many $D$-equivariant homotopy theories.

0.6. Simplicial diagrams of simplicial sets. Nothing new happens, and the same result hold as in discrete diagrams.

0.7. REMARK. It is very likely that the collection of model category structures on $S^{D}$ or Top ${ }^{D}$ described below (2) exhaust all possible such structures that extend the standard model category structure for simplicial sets considered as a subcategory of constant diagrams in $S^{D}$. Namely, one associates with every model category structure $M$ on $S^{D}$ a collection of orbits $O(M)$ defined by

$$
O(M)=\left\{X: D \rightarrow S \mid \operatorname{colim}_{D} X=(p t) \text {, and } X \text { is cofibrant in } M\right\} .
$$

Then it should be true that the model category structure associated with $O(M)$ is the given one.

0.8 . Small diagrams over large categories. The generalization discussed above $(0.3)$ allows us to define a model category structure on the category of all small functors $X: C \rightarrow$ (spaces) where now $C$ is any category, not necessarily small. A small functor (or small diagram) is one whose values on $C$ are determined by its values on a subset of obj $C$, via left Kan extensions; namely, $X(c)$ is the colimit over the appropriate over-category associated to $c$ and to a set of objects in $C$, that set may depend on $X$, of course.

It is easy to see that the free $C$ diagram $\{\operatorname{hom}(c,-)\}$ generated at $c \in$ obj $C$ is a small functor. Therefore, one can define a model category structure on small functors from $C$ to (spaces) using as orbits the collection of free orbits. This collection is easily seen to be locally small and to satisfy $\mathrm{Q} 0-\mathrm{Q} 3$ below.

As a prime example of the latter model category take $C=O_{D}$ where $O_{D}$ is the collection of all $D$-orbits

$$
O_{D}=\left\{T: D \rightarrow \text { spaces } \mid \text { colim }_{D} T=(p t)\right\} .
$$

This will yield a category of diagrams equivalent to $D$-diagrams with weak equivalences as in 0.2 or 0.5 and with the advantage that weak equivalences are now local weak equivalences and cofibrant objects are now retracts of free ones. This allows one to use the powerful methods of [6] and similar methods to the 'strong' model category structure on $D$-space since the category of $D$-spaces with the structure 0.5 or 1.3 below is equivalent to that of $O_{D}$-spaces with the 'local weak equivalence' as the weak equivalence.

We will not discuss here in any detail this category of small diagrams over large category. We intend to come back to it in a future paper. 
Similarly, one may wish to consider other collections of orbits to get various model category structures on $S^{D}$ or Top ${ }^{D}$.

0.9. REMARK. Working with small diagrams over large categories we can say that all the model category structures given in (0.5) above are but special cases of the canonical model category structure for diagrams of spaces given in [5]. This is so because for any given class of orbits $O$ in $S^{D}$ (or Top ${ }^{D}$ ) one considers small $O$-diagrams as a model category with weak equivalence being objectwise weak equivalence. Then the resulting homotopy theory on $S^{O}$ is equivalent to the homotopy theory on $S^{D}$ based on the collection of orbits $O$, using 0.8 above.

0.10 . Classification of diagrams of simplical sets. As a further application of 0.5 and 0.9 one can refine the classification result of [6]. In that paper $D$-diagrams of simplicial sets are classified, by means of function complexes, up to objectwise weak equivalence (0.1). Now one can use the same results to classify them up to the weak-equivalence of 0.5 : Let $\left\{O_{s}\right\}$ be any set of $D$-orbits, i.e. $D$-diagrams with $\operatorname{dirlim}_{D} O_{s}=(p t)$. Then the homotopy theory of $D$-diagrams with weak equivalence relation based on $\left\{O_{s}\right\}$ as in (0.5) is equivalent (as a simplicial model category) by $\S 2$ below and (2.2) of [5] to the "usual" homotopy theory of $\left\{O_{s}\right\}$-diagrams with objectwise weak equivalence; here $\left\{O_{s}\right\}$ denotes the full subcategory of $S^{D}$ with obj $O_{s}=\left\{O_{s}\right\}$. Therefore when one applies the Dwyer-Kan theory [6] to these $\left\{O_{s}\right\}$-diagrams one gets a much finer classification theory for $D$-diagram than the one given in [6].

0.11. Organization of the paper. The rest of this paper is organized as follows. In the first section we discuss the relation between model category structures on a simplicial category $\mathbf{M}$ and a large collection $\left\{O_{s}\right\}$ of orbits in $\mathbf{M}$. This is a direct generalization of $\$ 2$ of [5]. In the second and third section we discuss application to model category structures on the simplicial categories of diagrams of spaces $S^{D}$ and Top $^{D}$ and prove 0.2 above.

1. Generalized orbit categories. In [5] the authors give a general scheme for imposing a closed simplicial model category on simplicial categories (see Theorem 2.2 of [5]). We will freely use the results of that paper. In this section we want to note that their method works in a more general context that is needed in the next section. We are given a simplicial category $\mathbf{M}$ with a collection $O$ of objects of $\mathbf{M}$ to be called orbits for $\mathbf{M}$ if they satisfy certain axioms Q0-Q3 below. We assume that $\mathbf{M}$ is closed under all direct limits. Axioms Q0-Q3 say that each object in $O$ is "small" in the sense that taking its function complexes into certain diagrams commutes with taking the direct limit of these diagrams. The main result of [5] is that if $O$ is a set of orbits then there is a model category structure on $\mathbf{M}$ where weak equivalences (fibrations) are maps $f: X \rightarrow Y$ for which $\operatorname{hom}(T, f): \operatorname{hom}(T, X) \rightarrow \operatorname{hom}(T, Y)$ is a weak equivalence (fibration) for every $T \in O$.

Our main observation here is that the collection $O$ can be allowed to be large provided it is locally small in the following sense.

1.1. Definition. A full subcategory of objects $O=\left\{O_{e}\right\}_{e \in E} \subset \mathbf{M}$ is called locally small if for each object $X \in \mathbf{M}$ there exists a set $O(X)$ of objects in $O$ with the property that each $f: O_{e} \rightarrow X$ factors through a map $O_{f} \rightarrow X$ for some $O_{f} \in O(X)$. 
REMARK. This definition is motivated by a similar concept of André, [1].

1.2. The axioms for a collection $\left\{O_{e} \mid e \in E\right\}$ of orbits. Our axioms $\mathrm{Q} 0-\mathrm{Q} 3$ are all but identical to those of [5]. We reproduce them for convenience:

Q0. $\mathbf{M}$ is closed under arbitrary direct limits.

Q1. If

$$
\begin{array}{ccc}
O_{e} \otimes K & \rightarrow & O_{e} \otimes L \\
\downarrow & & \downarrow \\
X_{a} & \rightarrow & X_{a+1}
\end{array}
$$

is a push out diagram in $\mathbf{M}$ in which $L$ is a finite simplicial set, $K$ is a subcomplex of $L$ and $e \in E$, then, for every $e^{\prime} \in E$, the induced diagram in $\mathbf{S}$

$$
\begin{array}{ccc}
\operatorname{hom}\left(O_{e^{\prime}}, O_{e} \otimes K\right) & \rightarrow & \operatorname{hom}\left(O_{e^{\prime}}, O_{e} \otimes L\right) \\
\downarrow & & \downarrow \\
\operatorname{hom}\left(O_{e^{\prime}}, X_{a}\right) & \rightarrow & \operatorname{hom}\left(O_{e^{\prime}}, X_{a+1}\right)
\end{array}
$$

is up to homotopy (i.e., up to a weak equivalence) a push out diagram.

Q2. If $X_{q} \rightarrow \cdots \rightarrow X_{a} \rightarrow X_{a+1} \rightarrow \cdots$ is a (possibly transfinite) sequence of objects and maps in $\mathbf{M}$ such that each map $X_{a} \rightarrow X_{a+1}$ is induced as in Q1 and such that, for every limit ordinal $b$ involved, one has $X_{b}=\lim _{a<b} X_{a}$, then, for every $e \in E$, the induced map

$$
\lim _{a} \operatorname{hom}\left(O_{e}, X_{a}\right) \rightarrow \operatorname{hom}\left(O_{e}, \lim _{a} X_{a}\right) \in S
$$

is a weak equivalence.

Q3. There is a limit ordinal $c$ such that, for every sequence $X_{1} \rightarrow \cdots \rightarrow X_{a} \rightarrow$ $X_{a+1} \rightarrow \cdots$ as in Q2 which is indexed by the ordinals $<c$, one has

$$
\lim _{a} \operatorname{hom}\left(O_{e}, X_{a}\right)=\operatorname{hom}\left(O_{e}, \lim _{a} X_{a}\right) \text {. }
$$

We now say that a full subcategory of objects is a locally small category of orbits for $\mathbf{M}$ if $O$ is locally small in $\mathbf{M}$ and if $O$ satisfy axioms Q0-Q3 above.

The main result of this section is then

1.3. Proposition. The conclusion of Theorem 2.2 of [5] holds if one takes $O$ to be a locally small category of orbits for $M$; namely, $M$ admits a closed model category structure where a weak equivalence $f$ is a map with $f^{T}$ a weak equivalence for all $T \in O$, a fibration $f$ is a map with $f^{T}$ a fibration in $S$ for all $T \in O$ and the cofibrants are retracts of limits of towers as in $\mathrm{Q} 2$.

Proof. In fact the only thing that one needs to check is that the "small object argument" works under our assumptions $[8, \S 3$, p. 3.4]. In order to verify lifting and factorization properties we must check as in $[5,2.2]$ the right lifting property for the family of maps.

$$
T \otimes \Delta[n] \rightarrow T \otimes \Delta[n], \quad T \otimes V[n, k] \rightarrow T \otimes \Delta[n]
$$

where $T$ runs over all orbits. But this is reduced to checking a set of $T$ 's by

LEMMA. For any inclusion of simplicial sets $g: K \rightarrow L$ and for any map $f: X \rightarrow Y$ in $M$ there exists a set of orbits $O(f, g) \subset O$ so that for any map $\left(u_{x}, u_{y}\right)$ in the diagram 
below with $T \in O$ there is a factorization as indicated with $S \in O(f, g)$

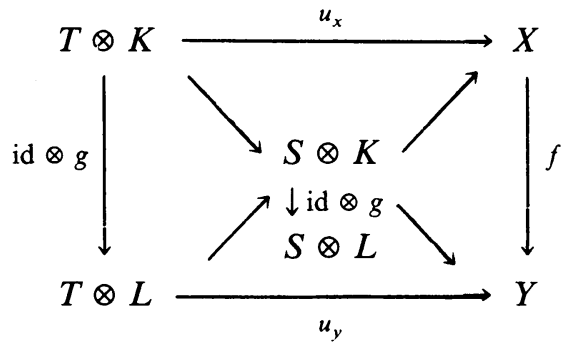

Proof. A map $\left(u_{x}, u_{y}\right)$ in the square above is uniquely given by a map of $T$ onto the function complexes in the outer square of the diagram:

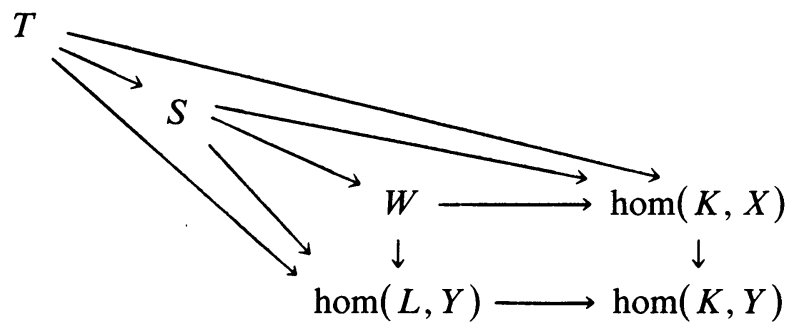

To factor such a map we need to construct $S$ above and the maps. Let $W$ be the pull-back. Take $O(f, g)=O(W)$ as in 1.1 above. Then the map $T \rightarrow W$ into the pull-back can be factored through an element $S \in O(W)=O(f, g)$; hence the result, since $W$ and $O(W)$ depend only on $f$ and $g$.

To continue the proof of 1.2 let us take for example the construction of factorization $f=p \circ i$ of a map $f: X \rightarrow Y$ in $\mathbf{M}$ as a cofibration followed by a trivial fibration. We follow [5 and 8, p. 3.2]. One constructs a factorization $X \stackrel{i}{\rightarrow} Z \stackrel{p}{\rightarrow} Y$ by taking $Z=\operatorname{dirlim} Z_{n}$ of a system $Z_{n-1} \rightarrow Z_{n}$ with $Z_{0}=X$. We define inductively $Z_{n}$ by the push out

$$
\begin{array}{ccc}
\coprod_{l \in A} \Delta\left[q_{e}\right] & \hookrightarrow & \coprod_{l \in A} \Delta\left[q_{e}\right] \\
\downarrow \amalg \alpha_{e} & & \downarrow \\
Z_{n-1} & \rightarrow & Z_{n}
\end{array}
$$

where $A$ is the set of all diagrams

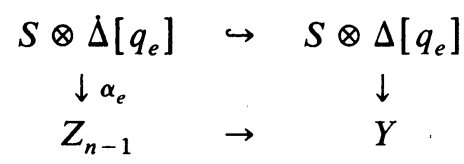

with $0 \leqslant q_{e}$ and $S$ any object in $O\left(p_{n-1}, g_{e}\right)$ with $g_{e} \Delta\left[q_{e}\right] \rightarrow \Delta\left[q_{e}\right]$ the standard inclusion, as in the Lemma above. Then one has a well-defined map $p_{n}: Z_{n} \rightarrow Y$ and one proceeds by induction. This gives factorization. Now to check the lifting property let $T$ be any orbit in $O$, then by Q0-Q3 any map $T \otimes \Delta[n] \rightarrow Z$ factors through some $Z_{n}$. But then by Lemma the map in the outer square below factors through some $S \otimes \Delta[n] \rightarrow S \otimes \Delta[n]$ for some $S \in O\left(p_{n}, g\right)$ 
$S \otimes \Delta[n] \rightarrow S \otimes \Delta[n]$ for some $S \in O\left(p_{n}, g\right)$

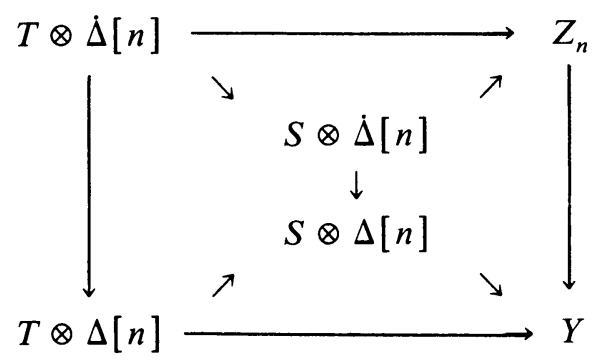

but this map of $S \otimes \Delta[n] \rightarrow S \otimes \Delta[n]$ was glued on to the next stage, so $T \otimes \Delta[n]$ lifts to $Z_{n+1}$, thus to $Z$ as needed. This shows that the argument in [5] works under our weaker assumptions.

2. Model-category structures for diagrams. We now want to apply 1.3 above in order to extend Quillen's model category structure on the category of topological spaces [8, II.3] to the category of $D$-diagrams of topological spaces. It is immediate that if $X, Y: D \rightarrow$ Top are $D$-diagram of topological spaces then $\operatorname{Hom}(X, Y)_{n}=$ $\operatorname{hom}\left(X \times \Delta^{n}, Y\right)$, with the natural simplicial operations, turns Top $^{D}$ into a simplicial category with $X \otimes K=X \times|K|$ and $X^{K}=X^{|K|}$ for any simplicial set $K$; here $\Delta^{n}$ denotes the geometric $n$-simplex $|\Delta[n]|$.

We prove 0.2 by choosing a class of orbits that induces via 1.3 above the desired model category structure. One takes the class of orbits for $D$, denoted by $O_{D}$, to be the class of all $D$-spaces $T$ with the property $\operatorname{dirlim}_{D} T=(p t)$, namely the class of all $D$-orbits. It is immediate that $O_{D}$ is locally small: If $X \in \operatorname{Top}^{D}$ and $f: T \rightarrow X$ is any map of $T \in O_{D}$ to $X$, then $\operatorname{dirlim} f: \operatorname{dirlim} T \rightarrow \operatorname{dirlim} X$ defines a point $t \in \operatorname{dirlim} X$; if $X_{t}$ is the orbit over $t$ then $f$ factors through the inclusion $X_{t} \rightarrow X$. Therefore, $O(X)=\left\{X_{t} \mid t \in \operatorname{dirlim} X\right\}$ will satisfy Definition 1.1. In order to prove axioms Q0-Q3 above we need

2.1. LEMMA. Let $X_{1} \rightarrow X_{2} \rightarrow \cdots \rightarrow X_{\alpha} \rightarrow X_{\alpha+1}$ be a possibly transfinite sequence as in axiom Q2 above. Then for all $X_{\alpha} \rightarrow X_{\beta}$ in the sequence one has a pull-back diagram:

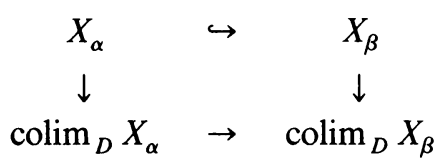

Proof. By definition, as a $D$-set $X_{\beta}$ is isomorphic to $X_{\alpha} \amalg Y$ for some $D$-set $Y$. Therefore, $X_{\alpha}$ is certainly the pull-back as a $D$-set. Hence the conclusion.

Lemma 2.1 allows us to construct an inverse $s$ to the natural map $j$ :

$$
j: \operatorname{dirlim}{ }_{\alpha} \operatorname{Hom}\left(T^{\prime}, X_{\alpha}\right) \rightarrow \operatorname{Hom}\left(T^{\prime}, \operatorname{dirlim} X_{\alpha}\right) .
$$

Given a simplex on the left $f: T^{\prime} \otimes \Delta^{n} \rightarrow \operatorname{dirlim} X_{\alpha}=X_{\infty}$, take dirlim to get a simplex $\tau$ in $\operatorname{dirlim}_{D} X_{\infty}$. But $\operatorname{dirlim}_{D} X_{\infty}=\operatorname{dirlim}_{\alpha}\left(\operatorname{dirlim}_{D} X_{\alpha}\right)$. Now dirlim ${ }_{D} X_{\alpha}$ is a sequence of C.W.-complexes relative to $\operatorname{dirlim}_{D} X_{1}$ and therefore any map of the compact spaces $\Delta^{n}$ to the limit space $X_{\infty}$ factors through $\operatorname{dirlim}_{D} X_{\gamma}$. Taking 
$X_{\gamma} \rightarrow X_{\infty}$ to be the map in 2.1 above we get a map $\tilde{f}: T^{\prime} \otimes \Delta^{n} \rightarrow X_{\gamma}$ by the property of pull-backs. So we define $s(f)$ to be the image of $\tilde{f}$ in $\operatorname{dirlim}{ }_{\alpha} \operatorname{Hom}\left(T^{\prime} \otimes \Delta^{n}, X_{\alpha}\right)$.

This shows that taking $\operatorname{Hom}($,$) commutes with taking direct limit over any$ sequence. The rest of axiom Q0-Q3 is proved similarly using 2.1: We leave it to the reader to check. This shows that Theorem 1.3 applies and we get a model category structure on $\operatorname{Top}^{D}$. To complete the proof of 0.2 we use [4]. It is shown there that every realization $|X|$ of a diagram of simplicial sets $X: D \rightarrow S$ is a transfinite direct limit as in Q2 above. Therefore $|X|$ is cofibrant as claimed. By 1.3 it is clearly also fibrant.

The proof of 0.5 in the case of $\mathbf{M}=S^{D}$ is very similar to the above. As a class of orbits one may take the colletion of objects in $S^{D}$ whose direct limit is $\Delta[0]$. This collection is clearly locally small. The proof proceeds just as in the topological case-using simplicial version of Lemma 2.1 above. Therefore taking $M=S^{D}$ in 1.3 and orbits to be all $D$ orbits we get from 1.3.

2.2 TheOREM. There is a closed model category structure on $S^{D}$ in which every $D$-space $T$ with $\lim _{D} T=\Delta[0]$ is cofibrant and $X$ is fibrant if $\operatorname{hom}_{D}(T, X)$ is a Kan complex for every such $T$.

2.3 REMARK. I suspect that every $X \in S$ is cofibrant, i.e. a retract of some transfinite limit as in Q2, in the model category structure (2.2) but I do not know how to prove it.

3. An application. Here we would like to indicate how the present result may be applied. The main direction we have in mind is for understanding the equivariant function complex for two $D$-spaces $\operatorname{map}_{D}(X, Y)=\operatorname{hom}_{D}(X, Y)$. For example, one would like to construct an (unstable) Adams spectral sequence starting with some homology theory on $D$-spaces and converging to the homotopy groups of these function complexes.

A moment of reflection will reveal that it is probably impossible to attempt an understanding of $\operatorname{hom}_{D}(X, Y)$ unless $X$ is a free $D$-space; namely, a cofibrant in the naive model category structure on Top ${ }^{D}$ and $S^{D}$ given in [5]. But most $D$-spaces are not cofibrant in that model category structure:

3.1. ExAmple. Let $D=J=(\cdot \rightarrow \cdot)$ as in 0.5 . Then a $J$-space is just a map of spaces $V \rightarrow W$. This $J$-space is cofibrant in the sense of [5] if and only if the map is a cofibration of spaces. If one naively converts a nonfibration $f: V \rightarrow W$ into a fibration $f: V \rightarrow M_{f} \sim W$ the function complex $\operatorname{map}_{J}\left(V \rightarrow W, V^{\prime} \rightarrow W^{\prime}\right)$ changes completely.

Now the meaning of the model category structure in $\S 2$, together with 1.3 above, is that every $D$-space $X$ can be modeled by some $O$-space $X^{0}$ where $O$ is a category of $D$-orbits, such that $X^{0}$ is a cofibrant $O$-space in the naive sense so that usual homological algebra and homotopical techniques of $[5,6]$ can be applied. In particular, the function complex $\operatorname{hom}_{D}(X, Y)$ is homotopically equivalent to a function complex hom ${ }_{D}\left(X^{0}, Y^{0}\right)$ where now $X^{0}$ is $O$-"free" or cofibrant, in the sense of Dwyer-Kan. As we remarked, such function complexes are much more accessible to the classical techniques than $\operatorname{hom}_{D}(X, Y)$ where $X$ is an arbitrary $D$-space. 
Let us then formulate this as follows [9]:

3.2. TheOREM. For $X$ in $\operatorname{Top}^{D}\left(\right.$ or $S^{D}$ ) there exist an $O$-space $X^{0}$ in $\operatorname{Top}^{O}\left(\right.$ or $S^{O}$ ) such that there is a weak equivalence of function complexes

$$
\operatorname{hom}_{D}(X, Y) \stackrel{\sim}{\rightarrow} \operatorname{hom}_{O}\left(X^{0}, Y^{0}\right) \quad \text { for any } Y \in \operatorname{Top}^{D}\left(S^{O}\right)
$$

and $X^{0}$ is cofibrant in the sense of Dwyer-Kan [5].

Proof. See [9].

\section{REFERENCES}

1. M. André, Méthode simpliciale en algèbre homologique et algèbre commutative, Lecture Notes in Math., vol. 32, Springer-Verlag, Berlin and New York, 1967.

2. G. Bredon, Equivariant cohomology theories, Lecture Notes in Math., vol. 34, Springer-Verlag, Berlin and New York, 1967.

3. E. Dror, Homology theory for diagrams of spaces, preprint (to appear).

4. E. Dror and A. Zabrodsky, Homotopy equivalences between diagrams of spaces, J. Pure Appl. Algebra (to appear).

5. W. G. Dwyer and D. M. Kan, Singular functors and realization functors, Proc. Kon. Nederl. Acad. 87 (2); Indag. Math. 46 (2) (1984).

6. _ A classification theorem for diagrams of simplicial sets, Topology 23 (1984), 139-155.

7. T. Gunnarsson, Abstract homotopy theory and related topics, Dept. of Math., Chalmers Univ. of Technology, Goteborg, Sweden, 1978.

8. D. G. Quillen, Homotopical algebra, Lecture Notes in Math., vol. 43, Springer-Verlag, Berlin and New York, 1967.

9. E. Dror Farjoun and A. Zabrodsky, The equivariant homotopy spectral sequence, Proc. Barcelona Conference 1986 (to appear).

Department of Mathematics, Hebrew University of JeruSAlem, JeruSAlem, ISRAel 